\title{
A haploproficient interaction of the transaldolase paralogue NQM1 with the transcription factor VHR1 affects stationary phase survival and oxidative stress resistance
}

Steve Michel ${ }^{1}$, Markus A Keller ${ }^{2}$, Mirjam MC Wamelink ${ }^{4}$ and Markus Ralser $2,3^{*}$

\begin{abstract}
Background: Studying the survival of yeast in stationary phase, known as chronological lifespan, led to the identification of molecular ageing factors conserved from yeast to higher organisms. To identify functional interactions among yeast chronological ageing genes, we conducted a haploproficiency screen on the basis of previously identified long-living mutants. For this, we created a library of heterozygous Saccharomyces cerevisiae double deletion strains and aged them in a competitive manner.

Results: Stationary phase survival was prolonged in a double heterozygous mutant of the metabolic enzyme non-quiescent mutant 1 (NQM1), a paralogue to the pentose phosphate pathway enzyme transaldolase (TAL1), and the transcription factor vitamin $\mathrm{H}$ response transcription factor 1 (VHR1). We find that cells deleted for the two genes possess increased clonogenicity at late stages of stationary phase survival, but find no indication that the mutations delay initial mortality upon reaching stationary phase, canonically defined as an extension of chronological lifespan. We show that both genes influence the concentration of metabolites of glycolysis and the pentose phosphate pathway, central metabolic players in the ageing process, and affect osmolality of growth media in stationary phase cultures. Moreover, NQM1 is glucose repressed and induced in a VHR1 dependent manner upon caloric restriction, on non-fermentable carbon sources, as well as under osmotic and oxidative stress. Finally, deletion of NQM1 is shown to confer resistance to oxidizing substances.
\end{abstract}

Conclusions: The transaldolase paralogue NQM1 and the transcription factor VHR1 interact haploproficiently and affect yeast stationary phase survival. The glucose repressed NQM1 gene is induced under various stress conditions, affects stress resistance and this process is dependent on VHR1. While NQM1 appears not to function in the pentose phosphate pathway, the interplay of NQM1 with VHR1 influences the yeast metabolic homeostasis and stress tolerance during stationary phase, processes associated with yeast ageing.

Keywords: Chronological lifespan, Pentose phosphate pathway, Transaldolase, NQM1, VHR1, Oxidative stress

\footnotetext{
* Correspondence: mr559@cam.ac.uk

${ }^{2}$ Department of Biochemistry and Cambridge Systems Biology Center,

University of Cambridge, 80, Tennis, Court Road, Cambridge CB2 1GA, UK

${ }^{3}$ MRC National Institute for Medical Research, The Ridgeway, Mill Hill,

London, UK

Full list of author information is available at the end of the article
} 


\section{Background}

Eukaryotic cells possess a variety of different strategies to maintain homeostasis, and adjust metabolism upon changes in environment and to survive stress situations such as oxido-reductive stress, osmotic turbulences, exposure to toxins, temperature imbalances or fluctuating nutrient concentrations [1-5]. This cellular flexibility is a requirement for a successful ageing process. It has thus become common view that cellular stress response mechanisms are key players in gerontology. Transcriptional regulation is important in cellular adaptation to both stress and ageing, and many stress responsive genes and regulatory systems have been identified [6-9]. The transcriptional response varies between stress conditions, but many gene sets and control mechanisms overlap and play a role in multiple situations. Key proteins required to establish the transcriptional stress response include the transcription factors Yap1p, Msn2/4p, Hsf1p, Hog1p/ Hot1p [10-12]. These transcriptional regulators allow the cell to react directly to the applied stress condition and induce effective defense mechanisms such as molecular chaperones and anti-oxidant proteins. However, transcriptional regulation does not explain the full spectra of cellular adaptations to changing environments and nutrition [13-15]. A presumably equally important, complementary strategy to react to environmental turbulences is to adjust cellular metabolism. A challenge for the cell in stress situations is to maintain the canonical function of metabolism, which is to continuously provide metabolites and cofactors such as ATP and NAD $(\mathrm{P}) \mathrm{H}$, required for the cell to survive and to continue proliferation upon the end of the stress situation. In particular, the ancient metabolic routes of glycolysis and the pentose phosphate pathway, amino acid and polyamine synthesis are at the centre of cellular metabolism, and have been shown to be involved in establishing fast and effective strategies in responding to situations like oxidative stress [4,5,16-18].

Genome duplication was one of the triggers that facilitated evolutionary adaptations to improve functionality of metabolism in stress situations. In budding yeast, a whole genome duplication event has been dated approximately 100 million years ago [19]. This created the basis for the evolution of isozymes that obtained specific functions during the stress response [20,21]. A diversification of enzyme function in stress situations has been reported for isozymes of isocitrate dehydrogenases [22,23], alcohol dehydrogenases [24], sirtuins [25,26], nutrient transporters [27] and glycolytic enzymes. The latter includes the most downstream glycolytic enzyme pyruvate kinase (isozymes PYK1 and PYK2), where the low-activity orthologue PYK2 is induced under conditions of high respiratory activity and protects the cells from oxidative damage [4].

Here we present evidence that NQM1 [28], a paralogue of the pentose phosphate pathway (PPP) enzyme transaldolase TAL1, as well as its regulating transcription factor VHR1 [29] are required during osmotic and oxidative stress, and affect survival of yeast cells upon long time starvation. We identified a heterozygous deletion of both genes through enrichment in a haploproficiency screen targeting genes previously implicated in chronological lifespan [30]. In subsequent analysis we find that NQM1 is induced upon nutrient limiting-, osmotic- and oxidative stress conditions and that this induction is dependent on the presence of VHR1. Finally, we show that the two genes cooperate in achieving oxidative stress tolerance of wild type cells.

\section{Methods}

\section{Yeast strains and cultivation}

All yeast strains used in this study are derived from the parent strain S288C. Wild type reference strains are haploid BY4741 (MAT a) or diploid BY4743 (MAT a/ MAT $\alpha)$ and were derived from the international knock out collection [31] obtained from EUROSCARF (Frankfurt, Germany; a complete list of deletion mutants used in this study is given as Additional file 1: Table S1).

Yeast was cultivated as detailed previously [32] at $30^{\circ} \mathrm{C}$ either in liquid or on solid (agar) yeast-extract peptone dextrose complex media (YPD: $20 \mathrm{~g} / \mathrm{l}$ peptone, $10 \mathrm{~g} / \mathrm{l}$ yeast extract, $20 \mathrm{~g} / \mathrm{l}$ agar, $2 \%$ glucose) or in synthetic complete media (SC: 6,8 g/l yeast nitrogen base with ammonium sulfate, $0.59 \mathrm{~g} / \mathrm{l}$ complete supplement mixture (CSM-ADEHIS-LEU-TRP-URA, MP Biomedicals), $10 \mathrm{mg} / \mathrm{ml}$ adenine, $20 \mathrm{mg} / \mathrm{ml}$ uracil, $20 \mathrm{mg} / \mathrm{ml}$ histidine, $60 \mathrm{mg} / \mathrm{ml}$ leucine, $40 \mathrm{mg} / \mathrm{ml}$ tryptophan, $20 \mathrm{~g} / \mathrm{l}$ agar) or synthetic media lacking the indicated amino acids or nucleobases. As sole carbon source, glucose $(2 \%, 0.5 \%, 0.05 \%)$, galactose $(2 \%)$, ethanol ( $3 \% / 0.1 \%$ glucose) or glycerol ( $3 \%$ / 0.1\% glucose) was used.

\section{Generation of a MAT a / MAT a heterozygous double gene deletion collection}

This study was conducted on the basis of results from the Kaeberlein laboratory that ranked yeast single gene knock-outs on the basis of their chronological lifespan [30]. From the 90 top scoring genes ranked, we crossed 82 MAT a with $85 M A T \alpha$ most long-living mutants that were present in our version of the library (Additional file 1: Table S1) and used them as basis to create a heterozygous KO library. In order to select for diploids and to facilitate differential barcode sequencing, the kanMX4 marker gene was replaced with LEU2 in the $82 M A T$ a strains via homologous recombination (Additional file 2: Table S2). For this, the LEU2 gene was amplified via PCR from plasmid pACT2 (Clontech, Table 1), the product was purified and transformed using the method described by Gietz et al. [33]. Positive transformants were selected on $\mathrm{SC}^{-\mathrm{LEU}}$ and 
Table 1 Plasmids used in the generation of mutant strains

\begin{tabular}{lll}
\hline Name & Source & Used for \\
\hline pACT2 & Clontech & Cloning of LEU2 \\
pAG25 & Euroscarf & Cloning of natMX4 \\
pAG32 & Euroscarf & Cloning of hphMX4 \\
\hline
\end{tabular}

correct integration of the marker through homologous recombination was verified in single clones via PCR.

The 82 marker-exchanged knock-out strains were then replicated into $9 \times 96$-well plates containing $50 \mu \mathrm{l}$ YPD, whereas $85 M A T \alpha$ knock-out strains were replicated into one 96-well plate containing $50 \mu \mathrm{l}$ YPD and incubated at $30^{\circ} \mathrm{C}$ for 24 hours. 10 MAT $\alpha$ strains were combined each and then transferred into replicates of the $M A T$ a plates. 9 plates with the MAT a / MAT $\alpha$ mixture were replicated on solid YPD media and incubated at $30^{\circ} \mathrm{C}$ for 2 days allowing mating to occur. Finally, diploids were selected on $\mathrm{SC}^{\text {-Leu }}+\mathrm{G}_{418}(800 \mu \mathrm{g} / \mathrm{ml})$ and subsequently pooled.

\section{Haploproficient screening and chronological ageing experiments}

Competitive haploproficiency screens were conducted by inoculating $100 \mathrm{ml}$ synthetic complete media (SC) $+2 \%$ glucose with overnight cultures of the wild type strain BY4743 and the library of heterozygous double mutants at an $\mathrm{OD}_{600}=0.3$ in a 1:1 ratio. Cultures were incubated at $30^{\circ} \mathrm{C}$ for 32 days on a shaking platform. Viability was determined every 2 nd to 4 th day by plating serial dilutions in triplicates onto SC media, allowing all cells to grow and onto $\mathrm{SC}^{\text {-Leu }}$, selecting for double mutants only. Colonies were counted and expressed as CFU/ml.

Non-competitive stationary phase survival experiments were conducted in quadruplicates by setting an overnight culture of the haploid strains to an $\mathrm{OD}_{600}=0.3$ in
$100 \mathrm{ml}$ SC media. Yeast was grown until stationary phase (2 days) and viability was recorded for 14 days.

\section{Isolation and sequencing of molecular barcodes from double heterozygous mutants}

The two barcode sequences in the double mutants were amplified via PCR from genomic DNA preparations as described previously [34] and sequenced by Sanger's didesoxynucleotide method [35]. The first barcode was amplified with the primers uptag/pFA6-rev (which binds within the kanMX4 marker, Table 2), whereas the second barcode was amplified with the primers LEU2-fwd/downtag (which binds within the LEU2 cassette, Table 2).

\section{Yeast cultivation and extraction for metabolomics analysis}

Sugar phosphate intermediates were extracted and quantified as described earlier [36]: Yeast strains were grown in synthetic complete media and were collected during logarithmic growth at an $\mathrm{OD}_{600}$ of $1.5 \pm 0.05$ using a rapid cold methanol quenching procedure [37]. Lysis of cells was achieved by FastPrep-24 cycles (MP Biomedicals, $3 \times$ $20 \mathrm{~s}, 6.5 \mathrm{~m} / \mathrm{s})$ in organic extraction buffer $(200 \mu \mathrm{l}$ of 75:25 acetonitrile:water containing $0.2 \%$ formic acid). After a second extraction step with the same amount of water the supernatants of both steps were combined, dried (SpeedVac concentrator), resuspended in $100 \mu \mathrm{l}$ water:acetonitrile (93:7) and then subjected to LC-MS/MS analysis. All solvents were used in HPLC grade or higher.

\section{LC-MS/MS measurements of sugar phosphates}

$1.5 \mu \mathrm{l}$ of the extracted samples were injected on a C8 column (Agilent Zorbax SB-C8 Rapid Resolution HD, $2.1 \times 100 \mathrm{~mm}, 1.8 \mu \mathrm{m}$ ) for separation with a gradient that started with isocratic flow at acetonitrile:water (12:88) for $3.5 \mathrm{~min}$, a subsequent ramp to $38: 62$ acetonitrile:

Table 2 Primer sequences used in strain generation, verification, sequencing

\begin{tabular}{|c|c|c|}
\hline Name & Used for & $5^{\prime}$ - 3' sequence \\
\hline kanMX4::LEU2 - fwd & Replace genomic kanMX4 with LEU2 (from pACT2) & $\begin{array}{l}\text { GCTGCAGGTCGACGGATCCCCGGGTTAAT TAAGG } \\
\text { CGCGCCAGATCAACTGTGGGAATACTCAGGT }\end{array}$ \\
\hline kanMX4:.LEU2 - rev & Replace genomic kanMX4 with LEU2 (from pACT2) & $\begin{array}{l}\text { ПTATTGTCAGTACTGATTAGAAAAACTCATCGAGCATC } \\
\text { AAATGATCATGATITCTGTTACACC }\end{array}$ \\
\hline LEU2 - fwd & Downtag amplification and sequencing in double mutants & AGGTGTAACAGAAAATCATGAT \\
\hline pFA6 - fwd & Downtag sequencing primer (kanMX4) & CTCGACATCATCTGCCCAGA \\
\hline pFA6 - rev & Uptag sequencing primer (kanMX4) & GTCTGCAGCGAGGAGCCGTA \\
\hline Uptag & Barcode forward primer & GATGTCCACGAGGTCTCT \\
\hline Downtag & Barcode reverse primer & CGGTGTCGGTCTCGTAG \\
\hline Tal1::HPH1-fwd & Replace genomic TAL 1 with hphMX4 (from pAG32) & $\begin{array}{l}\text { TAGTAAAATACTTCTCGAACTCGTCACATATACGTGTAC } \\
\text { ATAAGCTTGCCTTGTCCCCGCCG }\end{array}$ \\
\hline Tal1::HPH1-rev & Replace genomic TAL 1 with hphMX4 (from pAG32) & $\begin{array}{l}\text { GCATAAGGACATGGCCTAAATTAATATITCCGAGATAC } \\
\text { TTCCTCGACACTGGATGGCGGCGT }\end{array}$ \\
\hline NQM1::NTC-fwd & Replace genomic NQM1 with natMX4 (from pAG25) & CCATCTAGAATGGGGTGGACAACATATAAAAGAAGAG \\
\hline NQM1::NTC-rev & Replace genomic NQM1 with natMX4 (from pAG25) & TACGTCAGAATITTAATGAATATATAAGTCTGTACAC \\
\hline
\end{tabular}


water for $2.5 \mathrm{~min}$, an $30 \mathrm{sec}$ washing step at $42 \%$ acetonitrile, followed by re-equilibration for $30 \mathrm{sec}$. This totaled in a cycle time of $7.5 \mathrm{~min} .750 \mathrm{mg} / \mathrm{l}$ octylammonium acetate was added to all buffers as ion pairing reagent [38]. Analytes were quantified in MRM mode with an online coupled triple quadrupole mass spectrometer (Agilent 6460). Each metabolite was identified by similar retention time and fragmentation behavior and compared with externally measured commercially available standards. Optimal SRM transitions, ionization and fragmentation energies as well as ion source parameters can be found in [39]. Masshunter software (Agilent) was used for peak integration, external calibration (with repetitively measured standard dilution curves), absolute quantification and initial data analysis. All further data analysis was performed with R (http://www.r-project.org/).

\section{Determination of osmolality}

Osmolality was determined by freezing point depression in a Knauer Osmometer (Knauer) from aliquots of $1 \mathrm{ml}$ taken from the yeast cultures at the indicated time points throughout the chronological aging experiment. Yeast cultures were centrifuged twice at $16.000 \times \mathrm{g}$ for $2 \mathrm{~min} .200 \mu \mathrm{l}$ of the media were transferred to a vial and measured in triplicates.

\section{Stress tests}

Stress tests addressing oxidative stress tolerance (with menadione and $\left.\mathrm{H}_{2} \mathrm{O}_{2}\right)$, salt and osmotic stress $(\mathrm{NaCl}$, sorbitol) in liquid cultures or on solid plates were induced by adding the stressors to the given final concentration to the growth media as described previously [16].

\section{Quantitative RT-PCR}

Overnight cultures were diluted to an $\mathrm{OD}_{600}=0.15$ and grown until mid-log phase $\left(\mathrm{OD}_{600}=0.8-1.0\right)$ in $\mathrm{SC}+2 \%$ glucose (for menadione, $\mathrm{H}_{2} \mathrm{O}_{2}, \mathrm{NaCl}$, sorbitol treatment) and in $\mathrm{SC}$ with either $2 \%$ glucose, $2 \%$ galactose, $3 \%$ ethanol $+0.1 \%$ glucose or $3 \%$ glycerol $+0.1 \%$ glucose. In time course experiments (menadione, $\mathrm{H}_{2} \mathrm{O}_{2}, \mathrm{NaCl}$, sorbitol treatment), samples were taken on the specified time points.

For glucose restriction experiments, yeast was pregrown in triplicates until mid-log phase in $80 \mathrm{ml} \mathrm{SC}+$ $2 \%$ glucose media. Aliquots of $20 \mathrm{ml}$ were centrifuged at $2000 \times \mathrm{g}$ for $2 \mathrm{~min}$, and either frozen on dry-ice or transferred to media with low glucose concentration ( $0.5 \%$ or $0.05 \%)$ for $1 \mathrm{hr}$.

Total yeast RNA was isolated using the RNAeasy Mini Kit (Qiagen). Two $\mu$ g total yeast RNA was transcribed into cDNA using a 12-18 oligo dT primer and Moloney Murine Leukemia virus (M-MuLV) reverse transcriptase (NEB) according to the manufacturer's instructions. qRTPCR was performed on a Prism 7900HT cycler (Applied
Biosystems) with gene-specific primers (Table 3) and Maxima SYBR Green/Rox qPCR Master Mix (Thermo Scientific). The relative expression ratio of the target genes was normalized to the geometric mean of the three endogenous reference genes (UBA4, ATG27, TAF10) following the method of Pfaffl et al. [40].

\section{Results}

A haploproficient interaction of the transaldolase NQM1 and the transcription factor VHR1 enriches cells at a late stage of stationary phase survival

Haploinsufficiency and haploproficient interactions have been described as a measure of genetic interactions that identifies a different kind of gene dependency as compared to synthetic genetic or physical interactions [41-46]. Haploproficiency and haploinsufficiency are of prime physiological importance in diploid (and polyploid) organisms, as randomly occurring mutations more likely result in the loss of function of just one instead of all copies of a gene [47]. In order to identify haploproficient interactions in the context of cellular ageing, we focused on a set of genes previously associated with chronological lifespan in budding yeast [30]. We took the corresponding $82 M A T$ a and $85 M A T \alpha$ gene deletion strains as present in our copy of the yeast knock-out collection (Additional file 1: Table S1) and mated them with each other, adapting a multiple mating protocol established by Stelzl et al. [48]. The strategy in mating cells in repetitive batches of 10 strains facilitates a reasonable throughput, and avoids at the same time that a few strains possessing growth advantage can overgrow the library (Figure 1A).

In order to be able to select for diploids and to identify double heterozygous strains by means of barcode sequencing, we replaced the kanMX4 gene in all MAT a strains by the LEU2 marker (Figure 1A). After generation of the library containing up to 6970 bona fide dual heterozygous mutants, we inoculated this set of strains together with the diploid wild type BY4743. Viability of the population was followed for 32 days at $30^{\circ} \mathrm{C}$ by determining $\mathrm{CFU} / \mathrm{ml}$ by spotting serial dilutions on synthetic complete (SC) and SC media lacking leucine $\left(\mathrm{SC}^{-\mathrm{Leu}}\right)$ to distinguish the double mutants from the wild type cells (Figure 1B). This measure was used as indicator whether the population contained haplotypes with increased

Table 3 Primer sequences used in qRT-PCR

\begin{tabular}{lll}
\hline $\begin{array}{l}\text { Gene } \\
\text { product }\end{array}$ & Forward primer & Reverse primer \\
\hline NQM1 & GAAGTATGAACCACAGGATT & CAAAATCTTATCCATGGCGT \\
TAL1 & CCCATCATTGATCTTGGCTGC & GGCAGCTTGAATACCTTCCCA \\
UBA4 & AAGAGAGCAGCTTGCCAAGA & TCCAGCACCAACTACCAAAA \\
ATG27 & AGTTGTGCTTGGCGGAGTAT & CCTGCGAGAAGCATGATGTA \\
TAF10 & CCAGGATCAGGTCTTCCGTA & TGCTGTCCTTGCAATAGCTG \\
\hline
\end{tabular}


A)

MAT a strains: kanMX4 gene replaced with LEU2 gene

\begin{tabular}{r|c|c|}
\hline genomic DNA & LEU2 & genomic DNA \\
\hline uptag & downtag
\end{tabular}

MAT $\alpha$ strains: ORFs deleted by kanMX4 gene

\begin{tabular}{r|c|c|}
\hline genomic DNA & kanMX4 & genomic DNA \\
\hline uptag & downtag
\end{tabular}

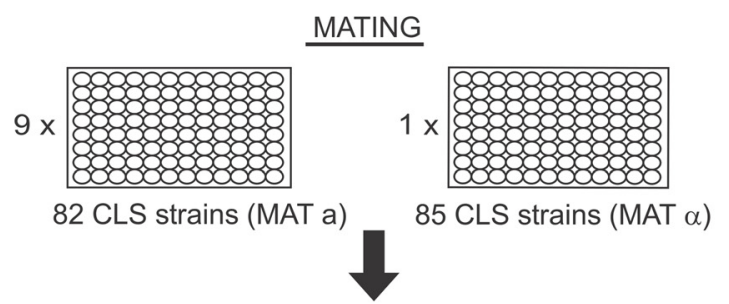

combine 10 MAT $\alpha$ strains

to 1 MAT a plate

2 days $130^{\circ} \mathrm{C}$

generates 6970 diploid, heterozygous double mutants

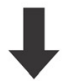

selection of diploid, heterozygous double mutants on SC - Leu / G418

B)

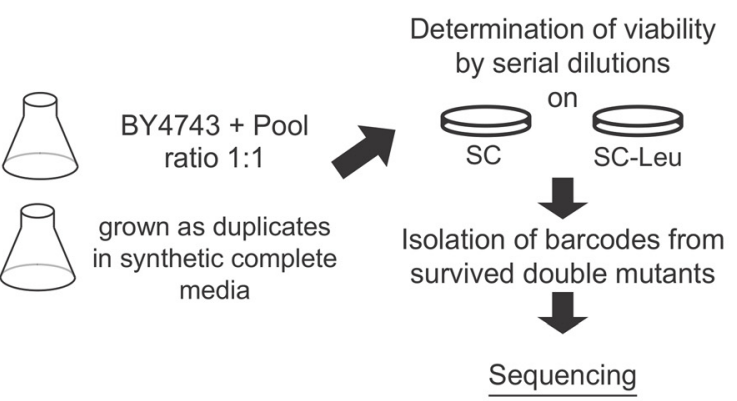

C)

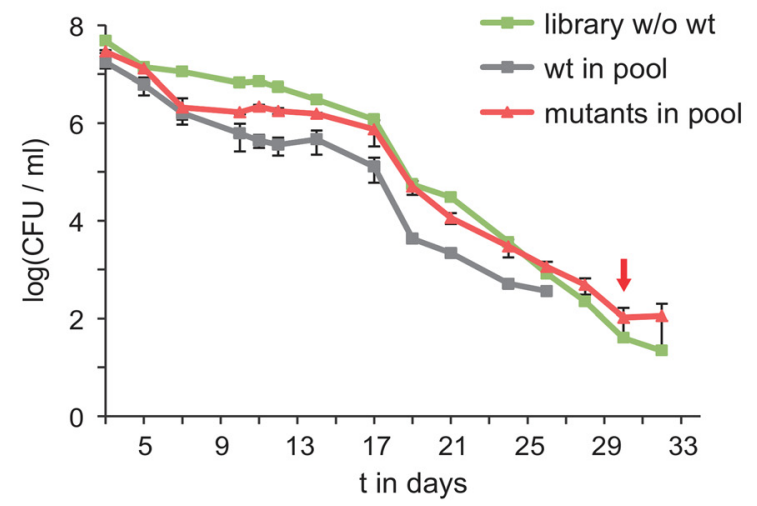

D)

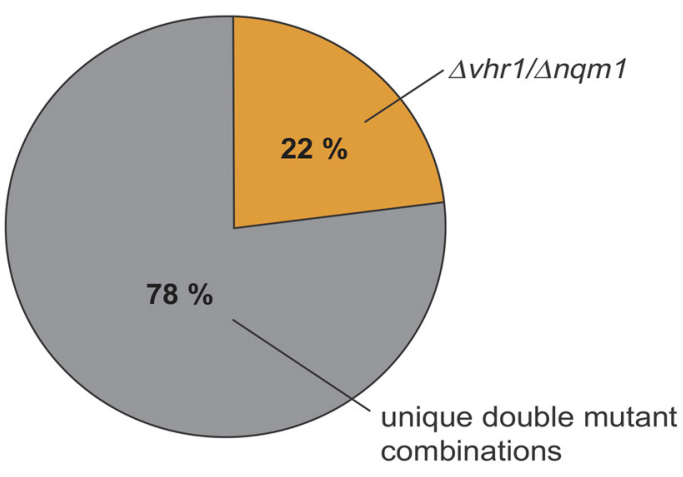

E)

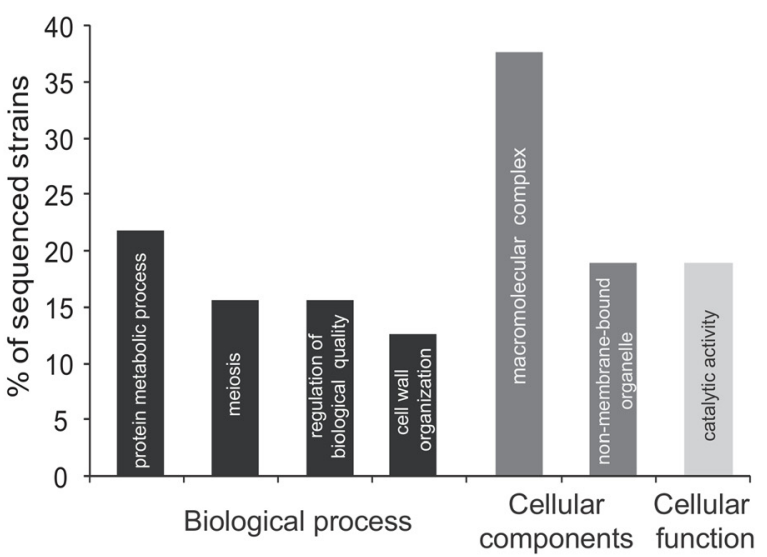

Figure 1 Haploproficiency of a VHR1/NQM1 double heterozygous mutant in late stationary phase. A) Generation of a heterozygous haplotype library. The kanMX4 marker present in the systematic knock-out strains was replaced by the LEU2 in 82 MAT a strains, and these were cross-mated in 96-well plates with the corresponding MAT a collection. After 2 days mating on YPD, double mutants were selected on synthetic media lacking leucine and supplemented with G418. B) The double heterozygous mutant library was combined with the wild type strain BY4743 in a 1:1 ratio and grown in independent duplicates at $30^{\circ} \mathrm{C}$ for 32 days in synthetic complete media (SC). Viability was monitored every 2 nd day by serial dilution plating C) Viability of cells during stationary phase in the double mutant library (green line), wild type within a competitive pool (grey line) or the mutants within the competitive pool (red line). The wild type lost viability in competitive pools at day 26 , the double mutants at day 32. The arrow marks time point of clonal selection. These trends in linear scaling are illustrated in Additional file 3, Figure S7, the trends of separate pools in Additional file 4: Figure S8. D) Identification of surviving haplotypes. 78\% of genotypes were unique, while the remaining cells were double-heterozygous for $\Delta v h r 1$ and $\Delta n q m 1$ E) Gene Ontology (GO) Term analyses of surviving double heterozygous yeast mutants on the basis of their single gene deletion. 
stationary phase survival (Figure 1C, and Additional file 3: Figure S7 (linear scaling), Additional file 4: Figure S8, (for trends in separate pools)). We observed a strong decrease in viability during the first days of stationary phase growth; with day 3 (Figure 1C) the cells reached a plateau phase lasting until the 18th day. Then, cells started to lose viability rapidly and nearly linearly until the 30th day. At day 26 , the wild type strain within the cultures had largely lost viability, but double mutants still produced viable colonies (Figure 1C). Thus, strains in the heterozygous library expressed prolonged stationary phase survival. From the surviving population, we randomly isolated 62 clones (31 from two parallel experiments). Via barcode sequencing [31], 39 haplotypes could be identified unambiguously which did lack two genes (Additional file 2: Table S2). 78\% of the isolated strains possessed a private (unique) genotype (Figure 1D). These deleted genes were enriched for (overlapping) Gene ontology (GO) process terms protein metabolic process, meiosis, regulation of biological activity, cell wall organization, cellular components terms macromolecular complex, non-membrane-bounded organelle and GO function term catalytic activity (Figure 1E) when considering the deletant genes as background set (Additional file 1: Table S1). The remaining cells $(22 \%)$ however corresponded to one sole enriched genotype. These strains were mainly isolated from one of the two experiments (Additional file 2: Table S2) and heterozygously deleted for the genes VHR1 and NQM1, encoding for a transcription factor and an alternative transaldolase.

\section{VHR1/NQM1 gene knock-outs possess increased} clonogenicity at a late stage of stationary phase survival We then tested whether this haploproficiency translates into altered survival in stationary phase of haploid cells.
We generated haploid $\Delta v h r 1, \Delta n q m 1$ as well as their double mutant $\Delta v h r 1 / \Delta n q m 1$ strains and monitored their survival in stationary phase. Overall, all haploid strains had a shorter chronological lifespan compared to the diploid strains. This appears to be attributable to the LEU2 marker, that had been repaired in the process of creating the diploid library [32]. The mortality during stationary phase, used to measure chronological lifespan, was similar among all mutant strains (Figure 2), and the initial clonogenicity on day three was similar among the strains (colony counts for wild type (51041667 CFUs/ml), $\Delta v h r 1$ (46083333 CFUs/ml), $\Delta n q m 1$ (48625000 CFUs/ml) and double mutant $\Delta v h r 1 / \Delta n q m 1$ (47708333 CFUs/ml). However, a significantly higher clonogenicity of the double mutant was detected at the last time point before stationary cultures lost viability ( $\mathrm{p}<0.05$, Figure 2 right panel). Vice versa, a decreased clonogenicity was detected for the single $\Delta v h r 1$ mutant $(\mathrm{p}<0.01)$. Thus, the haploproficient phenotype of the $v h r 1 / n q m 1$ double knock-out did correlate with a beneficial phenotype. Rather than a delayed decay in viability during the chronological ageing curve (known as chronological lifespan extension), the phenotype in both strains manifested as increased clonogenicity before stationary cultures lost survival.

\section{Altered concentration of glycolytic and PPP metabolites in $\Delta v h r 1$ and $\Delta n q m 1$ strains and in their double mutants} NQM1 is a paralogue of the transaldolase TAL1, an enzyme of the PPP [28]. We therefore tested whether $\Delta v h r 1, \Delta n q m 1$ and the double knock-outs $\Delta v h r 1 / \Delta n q m 1$ (Figure 3 left panel) as well as the corresponding heterozygous strains (Figure 3 right panel) exhibited differences in
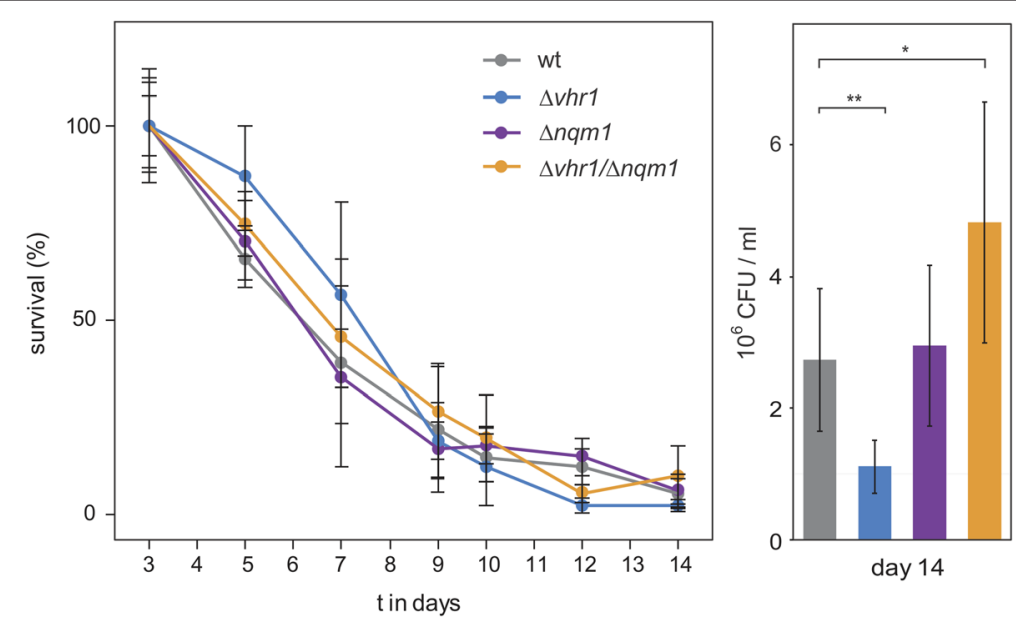

Figure 2 Chronological ageing of haploid $\Delta v \mathbf{h r} 1, \Delta n q m 1$ and $\Delta v \boldsymbol{h r} \mathbf{1} / \Delta n q m 1$ cells. Survival rates were determined in quadruplicate stationary cultures by spotting serial dilutions on synthetic complete media. Error bars +/- SD. Right panel: Number of colony forming cells on day 14 . The values represent the amount of cells that are able to form colonies (clonogenicity) on fresh agar media. Error bars +/- SEM. Student's t-test significance values $p<0.05={ }^{*}, p<0.01={ }^{* *}$. 


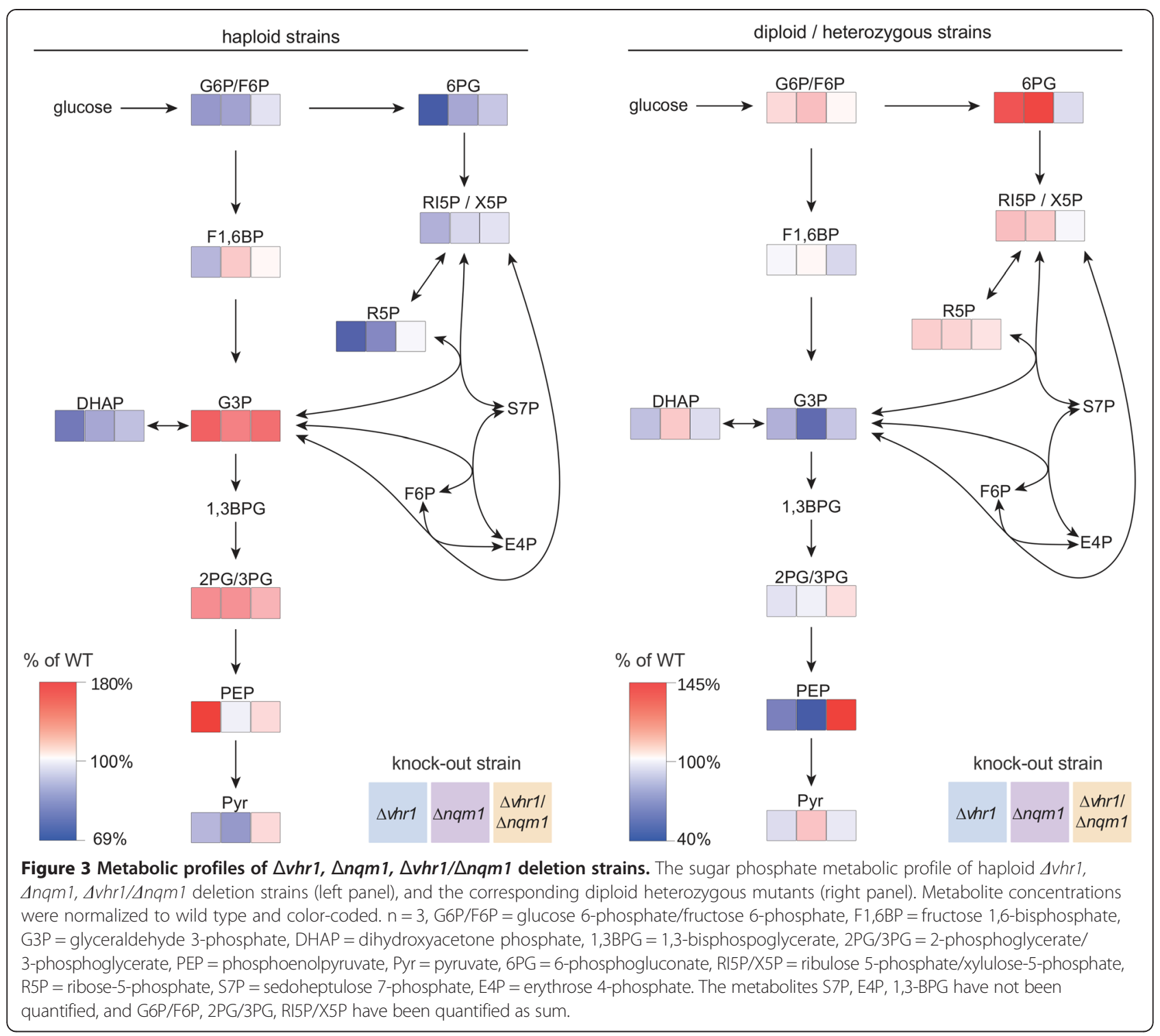

PPP intermediate metabolite concentrations. Liquid chromatography tandem mass spectrometry (LC-MS/MS) was used to profile the concentrations of intermediate metabolites as described earlier [39]. Indeed, we identified altered concentration of glycolytic and PPP metabolites in the mutant strains. The haploid $\Delta v h r 1$ strain exhibited an up to $43 \%$ decreased concentration of metabolites of upper glycolysis (glucose 6-phosphate/fructose 6-phosphate (G6P/F6P), fructose 1,6-bisphosphate (F1,6BP), dihydroxyacetone phosphate (DHAP) and the oxidative and partially non-oxidative part of the pentose phosphate pathway (6-phosphogluconate (6PG), ribulose 5-phosphate/xylulose 5-phosphate (RI5P/ $\mathrm{X} 5 \mathrm{P})$, ribose 5-phosphate (R5P)), and pyruvate (Pyr). In contrast, other metabolite levels of central and lower glycolysis (glyceraldehyde 3-phosphate (G3P), 2-phosphoglyerate/3- phosphoglycerate (2PG/3PG), phosphoenolpyruvate (PEP)) were elevated $65 \%$ above wild type level. The haploid $\Delta n q m 1$ strain displayed similar changes in metabolite levels as the $\Delta v h r 1$ mutant, but did not replicate the difference in PEP levels. The double mutant strain metabolic profile was less pronounced. Overall, its metabolite concentrations were more similar to the wild type strain (Figure 3).

Profiles of the heterozygous mutants were different to those of the haploid strains, but also here the metabolic profile of the double mutant was more similar to that of the wild type strain. Heterozygous deletion of VHR1 or NQM1 increased the concentration of PPP intermediates 6PG, RI5P/X5P and R5P, while VHR1/Dvhr1 NQM1/ $\Delta n q m 1$ yeast instead displayed nearly wild type level concentrations of the PPP intermediates (Figure 3, right 
A) Expression of NQM1 / TAL1 mRNA in wt, $\triangle n q m 1, \Delta t a l 1$ on different carbon sources and under glucose restriction
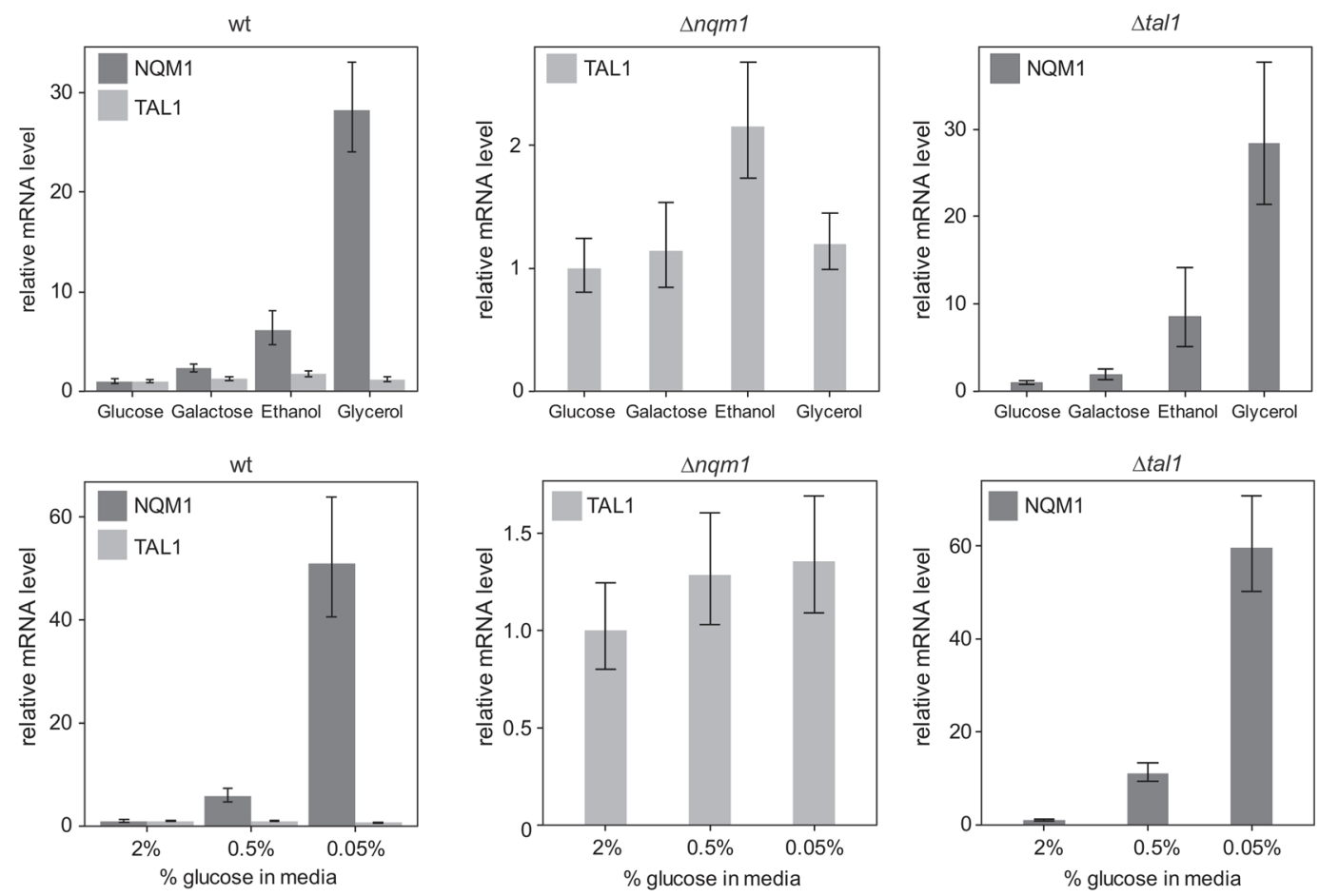

B) Expression of NQM1 / TAL1 mRNA in wt and $\triangle v h r 1$ on different carbon sources and under glucose restriction
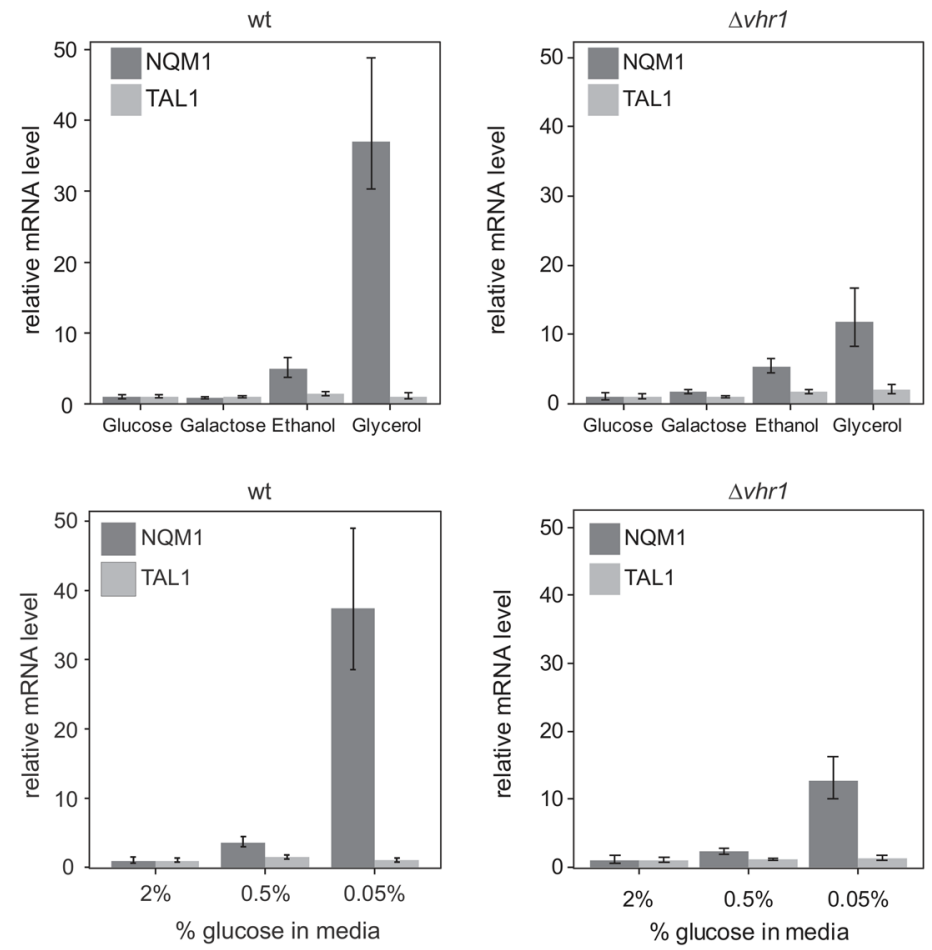

Figure 4 (See legend on next page.) 
(See figure on previous page.)

Figure 4 NQM1 mRNA levels are induced by caloric restriction and on non-fermentable carbon sources. A) Induction of NQM1 on non-fermentable carbon sources and calorie restriction in wild type, $\triangle$ nqm 1 and $\triangle$ tal1 deletion mutants. Expression of NQM1 mRNA levels is induced on non-fermentable carbon sources (ethanol, glycerol) and on galactose, indicating that NQM1 is subject to glucose repression. TAL 1 level instead are increased on ethanol only. B) Induction of NQM1 on non-fermentable carbon sources and calorie restriction in wild type and $\triangle$ vhr1 mutant. NQM1 induction is not suppressed by the deletion of $\Delta v h r 1$, but strongly reduced. Samples for qRT-PCR have been generated in liquid media containing the indicated carbon source. Cells were harvested at mid-log phase $\left(\mathrm{OD}_{600}=0.8-1.0\right)$. $n=3$, Error bars, $\pm \mathrm{SD}$.

panel). This effect was also observed for metabolites of lower glycolysis, where the double mutant did not replicate the concentration changes of both single mutants. Hence, while both the NQM1 and VHR1 deletions affected the concentration of glycolytic and PPP intermediates, the double deletion reversed some of the effects, and yielded opposite concentration effects in other cases (Figure 3).

\section{NQM1 is induced upon caloric restriction in a VHR1 dependent manner}

The PPP transaldolase Tal1p is a four-substrate enzyme, and required in the non - oxidative PPP to provide erythrose 4-phosphate and ribose 5-phoshate for the synthesis of amino acids and nucleotides, respectively [49,50]. While in vitro NQM1 is active on the same substrates, under normal laboratory growth conditions Tal1p is fully sufficient to maintain PPP activity while deletion of its isozyme Nqm1p has no apparent phenotype. Hence, it was suggested that Nqm1p could be important under other growth conditions [51]. Interestingly, a classic metabolic profile of a transaldolase knockout [17] was not observed in our metabolomics experiments, which indicates that NQM1 was not participating in the PPP, at least not in a comparable quantity as its paralogue TAL1. Thus, we investigated if mRNA expression of both transaldolase isozyme genes behaved similar upon changing nutrient supplementation by using qRT-PCR. For these experiments, wild type as well as $\Delta n q m 1$ and $\Delta$ tal1 yeast were grown in liquid media containing different carbon sources glucose, galactose, ethanol and glycerol. When cells were shifted towards the nonfermentable (gluconeogenetic) carbon sources ethanol or glycerol, NQM1 mRNA levels increased about 30-fold (Figure 4A). TAL1 expression levels instead did not change at the same level. TAL1 expression increased only on ethanol, and around 5-fold only (Figure 4A, upper panel). Interestingly, the induction of NQM1 was not affected by the deletion of $\triangle$ tal1, and the level of TAL1 not affected by the deletion $\Delta n q m 1$ (Figure $4 \mathrm{~A}$, middle and right panels). Hence, non - fermentable carbon sources induce the expression of NQM1. The induction of NQM1 was not influenced by presence or absence of its PPP isozyme TAL1, which in turn was not responsive to the carbon sources except a moderate induction on ethanol. As TAL1 is a highly active enzyme, this indicates that the gene expression regulation of NQM1 is not dependent on total transaldolase enzymatic activity.

The results instead implied that NQM1 could be a glucose repressed gene. We tested the effects of glucose caloric restriction on its expression levels. Upon lowering the media glucose concentration from $2 \%$ over $0.5 \%$ to $0.05 \%$ mRNA levels of NQM1 were induced up to 50 fold (Figure 4A, lower panels). In contrast, TAL1 mRNA levels were not affected by glucose restriction, and a $\Delta$ tal1 did not influence NQM1 expression. Thus, gene expression of paralogues is regulated independently from each other, and NQM1 but not TAL1, is a glucose repressed gene.

As we had identified NQM1 in concert with the transcription factor VHR1 (Figure 1), we next addressed their genetic interplay upon the nutritional shift. We altered carbon sources and glucose concentration in the $\Delta v h r 1$ mutant and monitored NQM1 levels in comparison to equally treated wild type cells. The absence of VHR1 did not prevent the NQM1 induction on non-fermentable carbon sources and upon glucose restriction. However, the expression levels of NQM1 reached only 1/4th of the level compared to wild type cells (Figure 4B). Hence, NQM1 is inducible on non - fermentable carbon sources and by glucose starvation. This function is independent to the canonical transaldolase TAL1, but requires the presence of the transcription factor $V H R 1$ for achieving wild-type expression levels.

\section{NQM1 is induced upon osmotic stress}

It has been reported that the anti-osmotic response of HOG1-HOT1, the major pathway of osmosis-controlled gene expression, affects the expression on NQM1 [12]. Northern blot analysis has shown that NQM1 is highly induced upon shift to $\mathrm{NaCl}$ in wild type cells, whereas a deletion in HOG1 diminished NQM1 expression more than $75 \%$ [12]. In order to test whether changes in osmotic potential are associated with the genetic interaction of NQM1 and VHR1, we followed media osmolality during chronological aging. These experiments were conducted in stationary phase wild type cells as well as in the $\Delta v h r 1$, $\Delta n q m 1$ and $\Delta v h r 1 / \Delta n q m 1$ mutants. The osmolality of blank media was determined at $233.75 \mathrm{mOsmol} / \mathrm{kg}$. Upon inoculating yeast, osmolality raised to $300-320 \mathrm{mOsmol} /$ 


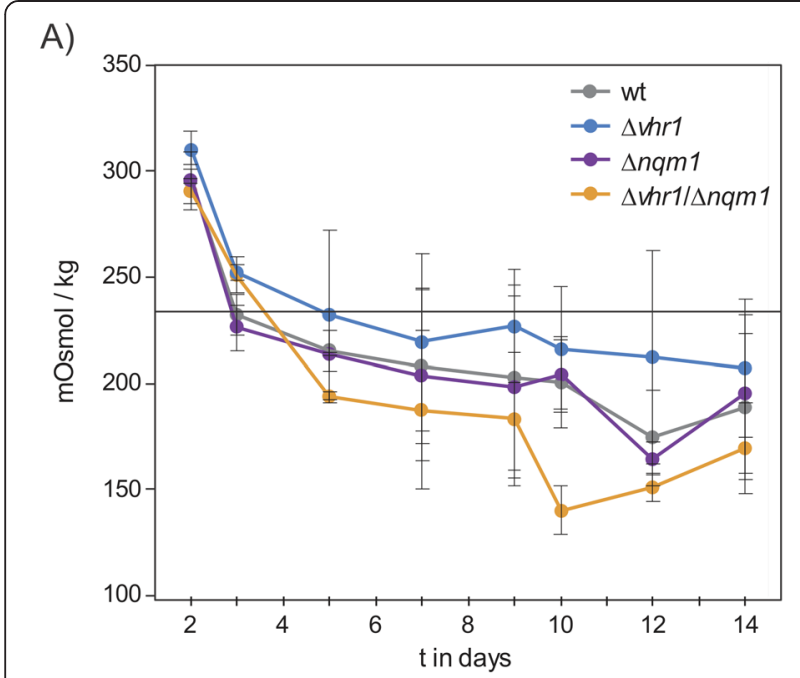

B)

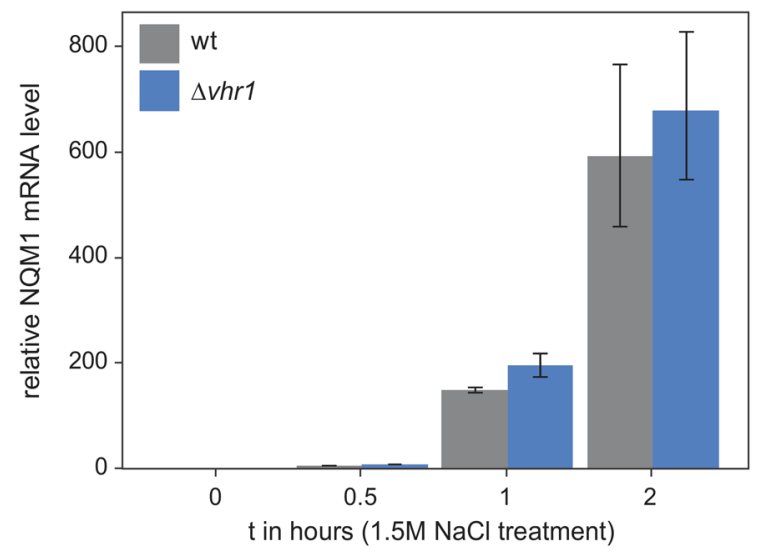

C)

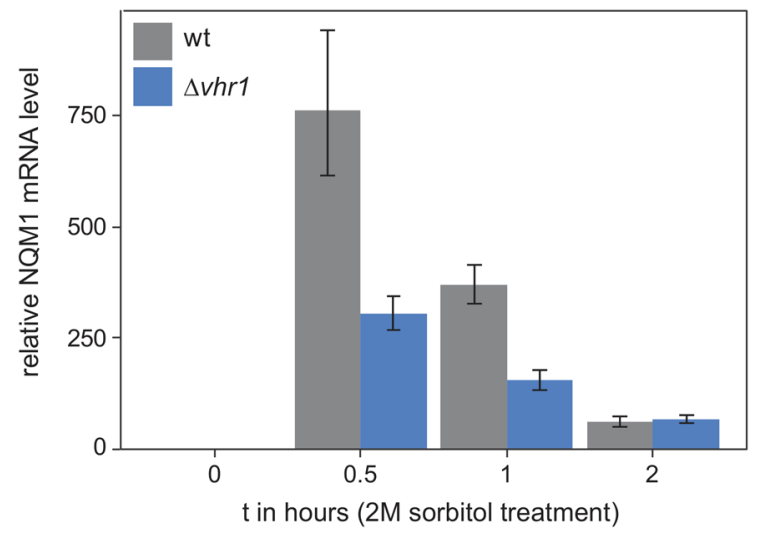

Figure 5 NQM1 mRNA expression is dependent on osmolarity.

A) Osmolality was determined in triplicates by freezing-point depression. The grey bar at $233.75 \mathrm{mOsmol} / \mathrm{kg}$ refers to the synthetic complete media osmolality prior to the experiment. All yeast strains depict a high osmolality at the start of the experiment which decreases during the chronological ageing below the control value (blank media) and remains stable until the end of the aging. Wild type (WT) and $\Delta$ nam 1 cells show a similar trend, whereas the decrease is stronger in the double mutant and diminished in $\triangle v h r 1$. B) + C) mRNA expression of NQM1 during the osmotic stress response in wild type and $\triangle \mathrm{v} h \mathrm{~h} 1$ mutant yeast. Time courses of NQM1 were generated by quantitative real-time-polymerase chain reaction (qRT-PCR) in wild type and $\Delta \mathrm{vhr} 1$ yeast treated with $\mathrm{NaCl}$ or sorbitol for $0,0.5,1$, and 2 hours. Upon $\mathrm{NaCl}$ treatment, NQM1 was severely induced in both wild type and $\triangle v$ hr 1 mutant yeast (Figure 5B). Upon sorbitol treatment, the induction pattern of NQM1 was similar in wild type and $\triangle v h r 1$ mutant yeast, albeit the magnitude in the $\Delta v h r 1$ mutant was reduced (Figure 5C). All experiments were performed in triplicates. Samples for qRT-PCR have been generated in liquid media containing glucose (2\%) as sole carbon source. $\mathrm{NaCl}$, sorbitol was added to induce osmotic shock upon cells reached mid-log phase $\left(\mathrm{OD}_{600}=0.8-1.0\right)$. Error bars, \pm SD.

$\mathrm{kg}$ within $48 \mathrm{hrs}$ indicating the release of yeast metabolites into the media. Afterwards, a slow but steady decline in osmolality until the end of the chronological lifespan was observed, which corresponds to a decline in media metabolite concentration. Osmolality decreased faster in the $\Delta v h r 1 / \Delta n q m 1$ double mutant, while in tendency slower in the $\Delta v h r 1$ mutant implicating metabolic differences between the strains (Figure 5A). Overall the osmolality did however not reach values which differed strongly to that of fresh yeast media. For this reason we consider it unlikely that difference in osmotic potential explains the different survival of $\Delta v h r 1 / \Delta n q m 1 \mathrm{mu}-$ tants. In agreement with this speculation, we did not detect altered resistance of $\Delta v h r 1$ and $\Delta n q m 1$ strains to $\mathrm{NaCl}$, sorbitol, $\mathrm{KCl}$ or $\mathrm{MnCl}_{2}$ that induces changes in osmolality (data not shown).

However, we could confirm that osmotic stressors induce NQM1 expression. We treated cells with $\mathrm{NaCl}$ and sorbitol, and consistent with the previous findings [12], NQM1 mRNA level were dramatically induced up to 750 -fold in the cells treated with $\mathrm{NaCl}$ and sorbitol (Figure 5B, C). The $\triangle v h r 1$ deletion did not significantly affect the NQM1 gene expression on $\mathrm{NaCl}$. On sorbitol however, a result analogous to glucose restriction was obtained: Deletion of VHR1 did not prevent the induction of $N Q M 1$, but in the absence of this transcription factor, NQM1 gene expression did not any longer reach wild type levels (Figure 5C).

\section{NQM1 limits oxidant tolerance}

Oxidative stress is a hallmark of ageing [52-56]. As cellular oxidant tolerance a direct consequence of their metabolic activity [16] we continued by testing for expression changes of NQM1 in cells treated with different concentrations of the oxidants menadione and hydrogen peroxide $\left(\mathrm{H}_{2} \mathrm{O}_{2}\right)$. 
NQM1 was strongly induced by menadione (25 - fold in wild type) and hydrogen peroxide (5.5 - fold in wild type). Deletion of VHR1 did not prevent the induction of NQM1 (Figure 6A, B). However, we detected higher expression of NQM1 in the $\Delta v h r 1$ strain treated with menadione (Figure 6A).

Then, we tested whether cells deficient in VHR1, NQM1 or TAL1 would exhibit altered resistance to oxidants or reductants. Stress resistance was assayed by serial dilution spot testing for survival of a set of single, double- and triple deletion mutants comprising the genes VHR1, NQM1, TAL1 on the oxidants diamide, $\mathrm{H}_{2} \mathrm{O}_{2}$, menadione, t-butyl hydroperoxide, cumene hydroperoxide and the reductants N-acetyl-cysteine, DTT, reduced glutathione. These experiments were conducted on media containing glucose, galactose, ethanol or glycerol. On glucose media, we identified a different tolerance of NQM1 and VHR1 mutants to menadione, $\mathrm{H}_{2} \mathrm{O}_{2}$ and diamide (Figure $6 \mathrm{C}$ ): $\Delta v h r 1$ and $\Delta v h r 1 / \Delta n q m 1$ cells were resistant to menadione, $\Delta n q m 1$ to $\mathrm{H}_{2} \mathrm{O}_{2}$, whereas the $\Delta v h r 1 / \Delta t a l 1$ mutant, the double transaldolase knock-out $\Delta n q m 1 / \Delta t a l 1$ and in particular the triple knock-out strain $\Delta v h r 1 / \Delta n q m 1 / \Delta t a l 1$ were sensitive to $\mathrm{H}_{2} \mathrm{O}_{2}$ (Figure $6 \mathrm{C}$ ). The response to the thiol-oxidizing substance diamide differed to peroxide stresses. $\Delta v h r 1$ and $\Delta v h r 1 / \Delta n q m 1$ yeast were sensitive to diamide, while all TAL1 mutants, $\Delta$ tal1, $\Delta n q m 1 / \Delta t a l 1$ and $\Delta v h r 1 / \Delta n q m 1 / \Delta t a l 1$ mutants were resistant. Stress tests with the corresponding diploid, heterozygous strains did not detect difference to the wild type (Additional file 5: Figure S9). Taken together, NQM1 is induced by oxidative stress in a partially VHR1 dependent manner. NQM1 and VHR1 mutants exhibit a series of stress resistance and sensitivity phenotypes. This indicates that the interplay of the two genes is required during a native antioxidant stress response.

\section{Discussion}

In this study we describe a competitive haploproficiency screening to identify genetic interactions that occur during yeast chronological ageing. We identified increased clonogenicity in late stationary phase of yeast mutants heterozygously deleted for $N Q M 1$ and VHR1, encoding for the yeast transaldolase paralogue (NQM1) and a transcription factor (VHR1). Since a competition experiment among 7000 double heterozygous deletion mutants reflects a complex system, we are limited to speculate about the dynamics that occurred during the course of the 30 day-long lasting stationary experiment. Previously, several studies showed that multiple factors like apoptosis [57], acetic acid accumulation [58] can affect the survival of yeast upon chronological aging. The latter could play a role in the selection dynamics of heterozygous mutants. Acetic acid has been proposed to be a major regulator of chronological lifespan [58] and has been linked to nutrient
[59] and Ras signaling [60]. This advantage of the heterozygous double mutant was however also confirmed in individual, haploid mutants. Also in these experiments, the phenotype did express as higher clonogenicity of the double mutant culture at late time-points, right before the stationary cultures lost viability. The phenotype did however not express as decreased mortality upon yeast entering stationary phase. It is thus possible that the double mutant maintained longer viability in stationary phase by being more efficient in adaptive re-growth [57]. We are thus not considering this phenotype as an increase in chronological lifespan in the canonical sense, but refer to it as increased survival at late stationary phase. Being a different phenotype to a chronological lifespan extension, this phenotype could however cover an alternative feature of cellular survival; the ability to persist nutrient limiting conditions is an essential feature in the natural environment, and can provide significant population advantages.

Recent studies suggested that NQM1 has either a direct role in gluconeogenic metabolism or in the supply of pentose phosphate precursors required for amino acid or nucleic acid biosynthesis under conditions under which regular synthesis from glucose 6-phosphate is difficult or insufficient [51]. Supporting parts of this assumption, we observed the induction of NQM1 on different carbon sources, caloric restriction and several stress conditions. NQM1 expression dominated over that of TAL1 on nonfermentable carbon sources (ethanol/glycerol), in the presence of osmotic stressors, and under caloric restriction (low glucose) conditions (the latter indicating that NQM1 is a glucose-repressed gene). However, we would like to note that NQM1 expression levels did not respond to the deletion of TAL1, and thus not to changes in total transaldolase and PPP activity. In addition, we did not find canonical transaldolase substrates to accumulate in $\Delta n q m 1$ cells, and the oxidant resistance profile of $\Delta t a l 1$ and $\Delta n q m 1$ strains were substantially different. Taken together these results indicate that despite its in vitro activity resembling Tal1p, NQM1 could have yet unknown physiological substrates, instead of participating in the PPP.

We found NQM1 induced under various stress conditions. This finding is consistent with a previous study focusing on the cumene hydroperoxide response, that identified a specific role of NQM1 in the cellular response to the oxidizing molecules [61] and that NQM1 is induced in those conditions [7]. The induction of NQM1 after release of glucose repression and in stress situations was in part dependent on the VHR1 transcription factor (Figures 4 and 5). The DNA binding motif of Vhr1p is well studied, and it has been shown that the transcription factor is able to recognize bZIP-like DNA motifs and Gcn4-like motifs [62]. We could however not detect such binding sites for the NQM1 locus using the Yeastract database, implying that the role of VHR1 in regulating 


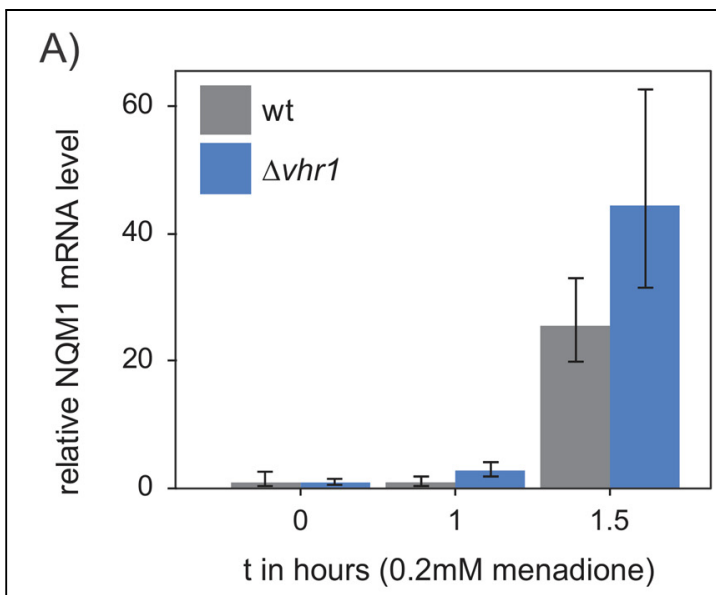

B)

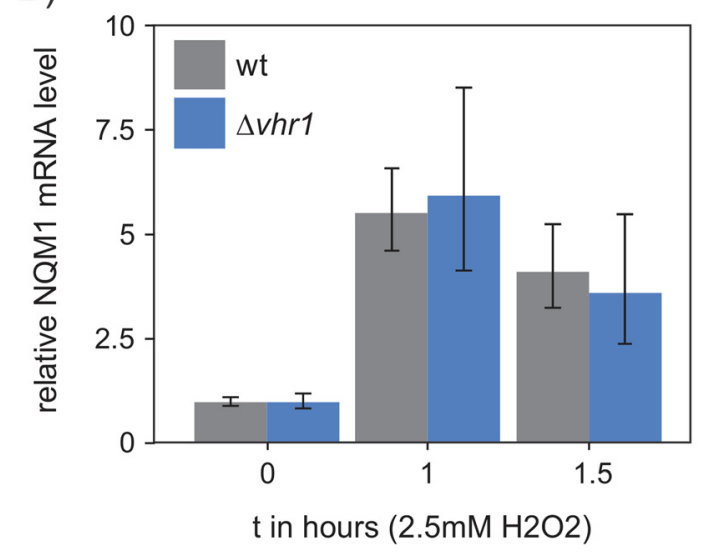

C)
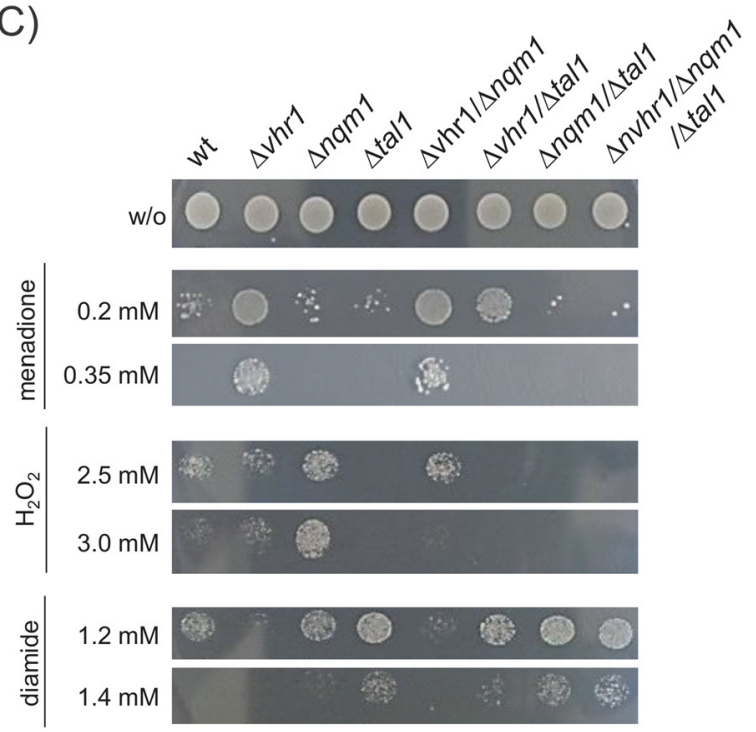

Figure 6 NQM1 Expression and growth phenotypes of NQM1/ VHR1 mutants under oxidative stress. A) NQM1 mRNA expression in response to treatment with the oxidant menadione. Wild type and $\triangle v$ hr1 mutant yeast induced NQM1 expression over time. B) $\mathrm{H}_{2} \mathrm{O}_{2}$ induces NQM1 in both wild type and $\triangle$ vhr1 mutant; qRT-PCR was conducted on cells grown in liquid media containing glucose (2\%) as sole carbon source. Oxidants were added to induce oxidative stress at mid-log growth phase $\left(\mathrm{OD}_{600}=0.8-1.0\right)$. C) Oxidant tolerance spot tests on media containing different concentrations of the oxidant menadione, $\mathrm{H}_{2} \mathrm{O}_{2}$ and diamide. $\Delta v h r 1$ and $\Delta v h r 1 / \Delta n q m 1$ mutants exhibit increased tolerance to menadione, $\Delta n q m 1$ to $\mathrm{H}_{2} \mathrm{O}_{2}$,

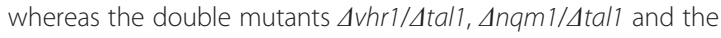
triple mutant $\Delta v \mathrm{hr} / \mathrm{\Delta nqm} \mathrm{l} / \Delta$ tall were sensitive to this oxidant. $\Delta v h r 1$ and $\Delta v h r 1 / \Delta n g m 1$ mutants were sensitive to diamide, while all $\Delta$ tal1 mutants were resistant to this oxidant.

NQM1 is likely indirect. Thus, further studies are needed to clarify the mechanisms and interactions which allow $V H R 1$ to influence the gene expression level of NQM1.

Yeast growth does change the growth media composition. The availability of nutrients, carbon or nitrogen sources will decline with time, while other metabolites (the exometabolome) accumulate [63-66]. Yeast preferentially metabolizes glucose through anaerobic metabolism resulting in the production of ethanol and carbon dioxide [67]. Changes in the concentration of soluble nutrients do affect the osmotic potential of the media. We detected a general decline in the media osmolality during in stationary phase survival (Figure 5A). Indicating that these changes reflect metabolic activity, this change in osmolality differed between wild type cells and the VHR1 and VHR1/NQM1 double mutants (Figure 5A). The changes in osmolality, albeit they were only moderate and thus unlikely a cause of toxicity, could however be recognized by mitogen activated protein (MAP) kinase pathways [68], in yeast represented by the high osmolarity glycerol (Hog1/Hot1) pathway [69]. NQM1 has previously been shown to be induced during osmotic shock and that this induction was dependent upon Hog1/Hot1 [12]. Our data is consistent with this observation; we find $N Q M 1$ strongly induced upon adding osmotic stressors (Figure 5B, C). Of note, the osmotic response that is triggered by Hoglp has been associated with increased lifespan by enhancing glycerol production from glycolytic intermediates [70]. The mechanisms how double heterozygosity of NQM1 and VHR1 influences late stationary phase survival could thus potentially dependent on the induction of NQM1 through osmotic signaling.

\section{Conclusions}

In summary, the data presented here shows that combined deficiency in NQM1 and VHR1 confers haploproficiency and extends clonogenicity at a late stage of yeast in stationary phase. We find that NQM1 is a glucose repressed and stress induced isoform of the pentose phosphate pathway transaldolase $T A L 1$, but is independently regulated and appears to have a 
different biochemical function compared to its paralogue. We find NQM1 to be induced under conditions of caloric restriction, osmotic stress and in the presence of some oxidative stressors, and that full induction of NQM1 requires the transcription factor VHR1. The detailed molecular mechanism by which NQM1 is implicated in stationary phase metabolism needs to be resolved in future studies. However, our data shows that NQM1 affects glucose metabolic activity in yeast stationary phase, under oxidative stress, osmotic stress and caloric restriction, and is thus implicated in difference facettes of the ageing process.

\section{Additional files}

Additional file 1: Table S1. Yeast strains used in the study.

Additional file 2: Table S2. Double mutants enriched in the competitive chronological lifespan experiments.

Additional file 3: Figure S7. Survival trend (\%) of the competitive chronological aging experiment. Viability of cells during competitive chronological aging shown for the wild types within the competitive pools (blue line - wt in pool1; green line - wt in pool 2) and the double mutants within the pools (red line - pool 1; purple line - pool 2).

Additional file 4: Figure S8. Trend of viability in competitive chronological aging. The non-competitive wild type (grey dotted line), the competitive double mutant library only (dark grey dotted line), the competitive pool 1 separated into wild type (red line) and mutants (orange line), the competitive pool 2 separated into wild type (green line) and mutants (blue line). The non-competitive wild type shows prolonged survival whereas upon competition against the double mutant library, the wild type lost viability prior to the mutants in both pools.

Additional file 5: Figure S9. Stress tolerance of haploid and diploid heterozygous deletion strains. A) Stress tolerance of stationary phase cultures. The haploid $\Delta v h r 1$ and the double mutant $\Delta v h r 1 / \Delta n q m 1$ show increased stress tolerance upon various oxidants on fermentable (glucose) and non-fermentable (ethanol) carbon sources. B) Stress tolerance of diploid, heterozygous deletion strains. The stress tolerance is comparable to the wild type strain.

\section{Competing interests}

The authors declare that they have no competing interests.

\section{Authors' contributions}

SM conducted yeast genetic experiments, RT-PCR and data analysis, and was involved in designing and conception of the study as well as in drafting the manuscript, MK and MW carried out the LC-MS/MS analyses and data analyses. MR conceived the study and wrote on the manuscript. All authors read and approved the final manuscript.

\section{Authors' information}

SM is a PhD student, MAK a postdoctoral fellow, MMW a staff scientist and MR a Principal investigator.

\section{Acknowledgements}

We thank the Max Planck Society, Wellcome Trust (RG 093735/Z/10/Z), the ERC (Starting grant 260809), and the Isaac Newton Trust for funding. Markus A Keller is supported by an Erwin Schroedinger postdoctoral fellowship (FWF, Austria, J 3341). Markus Ralser is a Wellcome Trust Research Career Development and Wellcome-Beit Prize fellow.

\section{Author details}

'Max Planck Institute for Molecular Genetics, Ihnestr 73, Berlin 14195, Germany. ${ }^{2}$ Department of Biochemistry and Cambridge Systems Biology Center, University of Cambridge, 80, Tennis, Court Road, Cambridge CB2 1GA, UK. ${ }^{3}$ MRC National Institute for Medical Research, The Ridgeway, Mill Hill, London, UK. ${ }^{4}$ Metabolic Unit, Department of Clinical Chemistry, VU University Medical Centre Amsterdam, Amsterdam, The Netherlands.
Received: 22 September 2014 Accepted: 21 January 2015

Published online: 11 February 2015

\section{References}

1. Simpson CE, Ashe MP. Adaptation to stress in yeast: to translate or not? Biochem Soc Trans. 2012;40:794-9.

2. Váchová L, Cáp M, Palková Z. Yeast colonies: a model for studies of aging, environmental adaptation, and longevity. Oxid Med Cell Longev. 2012;2012:601836. doi:10.1155/2012/601836. Epub 2012 Aug 13. Review. PubMed PMID: 22928081; PubMed Central PMCID: PMC3425895.

3. Bouwman J, Kiewiet J, Lindenbergh A, van Eunen K, Siderius M, Bakker BM. Metabolic regulation rather than de novo enzyme synthesis dominates the osmo-adaptation of yeast. Yeast. 2011;28(1):43-53. doi:10.1002/yea.1819. Epub 2010 Aug 27. PubMed PMID: 20803479.

4. Grüning N-M, Rinnerthaler M, Bluemlein $\mathrm{K}$, Mülleder $\mathrm{M}$, Wamelink MMC, Lehrach $\mathrm{H}$, et al. Pyruvate kinase triggers a metabolic feedback loop that controls redox metabolism in respiring cells. Cell Metab. 2011;14:415-27.

5. Krüger A, Vowinckel J, Mülleder M, Grote P, Capuano F, Bluemlein K, et al. Tpo 1-mediated spermine and spermidine export controls cell cycle delay and times antioxidant protein expression during the oxidative stress response. EMBO Rep. 2013;14:1113-9.

6. De Nadal E, Ammerer G, Posas F. Controlling gene expression in response to stress. Nat Rev Genet. 2011;12:833-45.

7. Gasch AP, Spellman PT, Kao CM, Carmel-Harel O, Eisen MB, Storz G, et al. Genomic expression programs in the response of yeast cells to environmental changes. Mol Biol Cell. 2000;11:4241-57.

8. Gasch AP, Werner-Washburne M. The genomics of yeast responses to environmental stress and starvation. Funct Integr Genomics. 2002;2:181-92

9. Gasch AP. Comparative genomics of the environmental stress response in ascomycete fungi. Yeast. 2007;24(11):961-76. Review. PubMed PMID: 17605132

10. Marinho HS, Real C, Cyrne L, Soares H, Antunes F. Hydrogen peroxide sensing, signaling and regulation of transcription factors. Redox Biol. 2014;2:535-62.

11. Capaldi AP, Kaplan T, Liu Y, Habib N, Regev A, Friedman N, et al. Structure and function of a transcriptional network activated by the MAPK Hog1. Nat Genet. 2008;40:1300-6.

12. Rep M. The transcriptional response of saccharomyces cerevisiae to osmotic shock. hot $1 \mathrm{p}$ and $\mathrm{msn} 2 \mathrm{p} / \mathrm{msn} 4 \mathrm{p}$ are required for the induction of subsets of high osmolarity glycerol pathway-dependent genes. J Biol Chem. 2000;275:8290-300

13. Ewald JC, Matt T, Zamboni N. The integrated response of primary metabolites to gene deletions and the environment. Mol Biosyst. 2013;9:440-6.

14. Buescher JM, Liebermeister W, Jules M, Uhr M, Muntel J, Botella E, et al. Global network reorganization during dynamic adaptations of Bacillus subtilis metabolism. Science. 2012;335:1099-103.

15. Grüning N-M, Lehrach H, Ralser M. Regulatory crosstalk of the metabolic network. Trends Biochem Sci. 2010;35:220-7.

16. Ralser M, Wamelink MM, Kowald A, Gerisch B, Heeren G, Struys EA, et al. Dynamic rerouting of the carbohydrate flux is key to counteracting oxidative stress. J Biol. 2007:6:10

17. Krüger A, Grüning N-M, Wamelink MMC, Kerick M, Kirpy A, Parkhomchuk D, et al. The pentose phosphate pathway is a metabolic redox sensor and regulates transcription during the antioxidant response. Antioxid Redox Signal. 2011;15:311-24

18. Harding HP, Zhang Y, Zeng H, Novoa I, Lu PD, Calfon M, et al. An integrated stress response regulates amino acid metabolism and resistance to oxidative stress. Mol Cell. 2003:11(3):619-33. PubMed PMID: 12667446.

19. Turunen $\mathrm{O}$, Seelke $R$, Macosko J. In silico evidence for functional specialization after genome duplication in yeast. FEMS Yeast Res. 2009:9:16-31.

20. Scharte J, Schön H, Tjaden Z, Weis E, von Schaewen A. Isoenzyme replacement of glucose-6-phosphate dehydrogenase in the cytosol improves stress tolerance in plants. Proc Natl Acad Sci U S A. 2009;106:8061-6.

21. Jang HH, Lee KO, Chi YH, Jung BG, Park SK, Park JH, et al. Two enzymes in one; two yeast peroxiredoxins display oxidative stress-dependent switching from a peroxidase to a molecular chaperone function. Cell. 2004;117:625-35.

22. Haselbeck RJ, McAlister-Henn L. Function and expression of yeast mitochondrial NAD- and NADP-specific isocitrate dehydrogenases. J Biol Chem. 1993:268:12116-22.

23. Henke B, Girzalsky W, Berteaux-Lecellier V, Erdmann R. IDP3 encodes a peroxisomal NADP-dependent isocitrate dehydrogenase required for the beta-oxidation of unsaturated fatty acids. J Biol Chem. 1998;273:3702-11. 
24. De Smidt O, du Preez JC, Albertyn J. The alcohol dehydrogenases of Saccharomyces cerevisiae: a comprehensive review. FEMS Yeast Res. 2008:8:967-78

25. LiX, Kazgan N. Mammalian sirtuins and energy metabolism. Int J Biol Sci. 2011;7:575-87.

26. Lu S-P, Lin S-J. Regulation of yeast sirtuins by NAD(+) metabolism and calorie restriction. Biochim Biophys Acta. 1804:2010:1567-75.

27. Kim J-H, Roy A, Jouandot D, Cho KH. The glucose signaling network in yeast. Biochim Biophys Acta. 1830;2013:5204-10.

28. Huang $\mathrm{H}$, Rong $\mathrm{H}$, Li X, Tong S, Zhu Z, Niu L, et al. The crystal structure and identification of NQM1/YGR043C, a transaldolase from Saccharomyces cerevisiae. Proteins. 2008;73(4):1076-81. doi:10.1002/prot.22237. PubMed PMID: 18831051

29. Weider M, Machnik A, Klebl F, Sauer N. Vhr1p, a new transcription factor from budding yeast, regulates biotin-dependent expression of VHT1 and BIO5. J Biol Chem. 2006;281:13513-24.

30. Powers RW, Kaeberlein M, Caldwell SD, Kennedy BK, Fields S. Extension of chronological life span in yeast by decreased TOR pathway signaling. Mol Cell Biol. 2006;16:174-84

31. Winzeler EA. Functional characterization of the $\mathrm{s}$. cerevisiae genome by gene deletion and parallel analysis. Science (80- ). 1999;285:901-6.

32. Mülleder M, Capuano F, Pir P, Christen S, Sauer U, Oliver SG, et al. A prototrophic deletion mutant collection for yeast metabolomics and systems biology. Nat Biotechnol. 2012;30:1176-8.

33. Gietz RD, Woods RA. Yeast transformation by the LiAc/SS Carrier DNA/PEG method. Methods Mol Biol. 2006:313:107-20. PubMed PMID: 16118429.

34. Harju S, Fedosyuk H, Peterson KR. Rapid isolation of yeast genomic DNA: bust n' grab. BMC Biotechnol. 2004;4:8.

35. Sanger F, Nicklen S. DNA sequencing with chain-terminating. Proc Natl Acad Sci U S A. 1977;74:5463-7.

36. Grüning NM, Du D, Keller MA, Luisi BF, Ralser M. Inhibition of triosephosphate isomerase by phosphoenolpyruvate in the feedbackregulation of glycolysis. Open Biol. 2014;4:130232. doi:10.1098/rsob.130232. PubMed PMID: 24598263; PubMed Central PMCID: PMC3971408.

37. de Koning W, van Dam K. A method for the determination of changes of glycolytic metabolites in yeast on a subsecond time scale using extraction at neutral pH. Anal Biochem. 1992;204(1):118-23. PubMed PMID: 1514678.

38. Wamelink MMC, Struys EA, Huck JHJ, Roos B, van der Knaap MS, Jakobs C, et al. Quantification of sugar phosphate intermediates of the pentose phosphate pathway by LC-MS/MS: application to two new inherited defects of metabolism. J Chromatogr B Analyt Technol Biomed Life Sci. 2005;823:18-25

39. Keller MA, Turchyn AV, Ralser M. Non-enzymatic glycolysis and pentose phosphate pathway-like reactions in a plausible Archean ocean. Mol Syst Biol. 2014;10:725

40. Pfaffl MW. A new mathematical model for relative quantification in real-time RT-PCR. Nucleic Acids Res. 2001;29:e45.

41. Giaever G, Shoemaker DD, Jones TW, Liang H, Winzeler EA, Astromoff A, et al. Genomic profiling of drug sensitivities via induced haploinsufficiency. Nat Genet. 1999;21:278-83.

42. Giaever G, Flaherty P, Kumm J, Proctor M, Nislow C, Jaramillo DF, et al. Chemogenomic profiling: identifying the functional interactions of small molecules in yeast. Proc Natl Acad Sci U S A. 2004;101:793-8.

43. Lum PY, Armour CD, Stepaniants SB, Cavet G, Wolf MK, Butler JS, et al. Discovering modes of action for therapeutic compounds using a genome-wide screen of yeast heterozygotes. Cell. 2004;116:121-37.

44. Delneri D, Hoyle DC, Gkargkas K, Cross EMM, Rash B, Zeef L, et al. Identification and characterization of high-flux-control genes of yeast through competition analyses in continuous cultures. Nat Genet. 2008:40:113-7.

45. Pir P, Gutteridge A, Wu J, Rash B, Kell DB, Zhang N, et al. The genetic control of growth rate: a systems biology study in yeast. BMC Syst Biol. 2012;6:4.

46. De Clare M, Oliver SG. Copy-number variation of cancer-gene orthologs is sufficient to induce cancer-like symptoms in Saccharomyces cerevisiae. BMC Biol. 2013;11:24.

47. Korona R. Experimental studies of deleterious mutation in Saccharomyces cerevisiae. Res Microbiol. 2004;155:301-10.

48. Stelzl U, Worm U, Lalowski M, Haenig C, Brembeck FH, Goehler H, et al. A human protein-protein interaction network: a resource for annotating the proteome. Cell. 2005;122:957-68.

49. Schaaff I, Hohmann S, Zimmermann FK. Molecular analysis of the structural gene for yeast transaldolase. Eur J Biochem. 1990;188:597-603.

50. Jacoby J, Hollenberg CP, Heinisch JJ. Transaldolase mutants in the yeast Kluyveromyces lactis provide evidence that glucose can be metabolized through the pentose phosphate pathway. Mol Microbiol. 1993;10(4):867-76. PubMed PMID: 7934848

51. Costenoble R, Picotti P, Reiter L, Stallmach R, Heinemann M, Sauer U, et al. Comprehensive quantitative analysis of central carbon and amino-acid metabolism in Saccharomyces cerevisiae under multiple conditions by targeted proteomics. Mol Syst Biol. 2011;7:464.

52. Longo VD, Shadel GS, Kaeberlein M, Kennedy B. Replicative and chronological aging in Saccharomyces cerevisiae. Cell Metab. 2012;16:18-31.

53. Kang WK, Kim YH, Kim B-S, Kim J-Y. Growth phase-dependent roles of Sir2 in oxidative stress resistance and chronological lifespan in yeast. J Microbiol. 2014:52:652-8

54. Muid KA, Karakaya HÇ, Koc A. Absence of superoxide dismutase activity causes nuclear DNA fragmentation during the aging process. Biochem Biophys Res Commun. 2014;444:260-3.

55. Choi J-S, Lee C-K. Maintenance of cellular ATP level by caloric restriction correlates chronological survival of budding yeast. Biochem Biophys Res Commun. 2013:439:126-31.

56. Merksamer PI, Liu Y, He W, Hirschey MD, Chen D, Verdin E. The sirtuins, oxidative stress and aging: an emerging link. Aging (Albany NY). 2013:5:144-50

57. Fabrizio P, Battistella L, Vardavas R, Gattazzo C, Liou L-L, Diaspro A, et al. Superoxide is a mediator of an altruistic aging program in Saccharomyces cerevisiae. J Cell Biol. 2004;166:1055-67.

58. Burtner CR, Murakami CJ, Kennedy BK, Kaeberlein M. A molecular mechanism of chronological aging in yeast. Cell Cycle. 2009;8:1256-70

59. Almeida B, Ohlmeier S, Almeida AJ, Madeo F, Leão C, Rodrigues F, et al. Yeast protein expression profile during acetic acid-induced apoptosis indicates causal involvement of the TOR pathway. Proteomics. 2009;9:720-32.

60. Colombo S, Ma P, Cauwenberg L, Winderickx J, Crauwels M, Teunissen A, et al. Involvement of distinct G-proteins, Gpa2 and Ras, in glucose- and intracellular acidification-induced CAMP signalling in the yeast Saccharomyces cerevisiae. EMBO J. 1998;17:3326-41.

61. Sha W, Martins AM, Laubenbacher R, Mendes P, Shulaev V. The genomewide early temporal response of Saccharomyces cerevisiae to oxidative stress induced by cumene hydroperoxide. PLoS One. 2013;8:e74939.

62. Gordân R, Murphy KF, Mccord RP, Zhu C, Vedenko A, Bulyk ML. Curated collection of yeast transcription factor DNA binding specificity data reveals novel structural and gene regulatory insights. Genome Biol. 2011;12:R125.

63. Paczia N, Nilgen A, Lehmann T, Gätgens J, Wiechert W, Noack S. Extensive exometabolome analysis reveals extended overflow metabolism in various microorganisms. Microb Cell Fact. 2012;11:122.

64. Kell DB, Brown M, Davey HM, Dunn WB, Spasic I, Oliver SG. Metabolic footprinting and systems biology: the medium is the message. Nat Rev Microbiol. 2005:3:557-65.

65. Castrillo Il, Zeef LA, Hoyle DC, Zhang N, Hayes A, Gardner DC, et al. Growth control of the eukaryote cell: a systems biology study in yeast. J Biol. 2007;6(2):4. PubMed PMID: 17439666; PubMed Central PMCID: PMC2373899.

66. Gutteridge A, Pir P, Castrillo J, Charles PD, Lilley KS, Oliver SG. Nutrient control of eukaryote cell growth: a systems biology study in yeast. BMC Biol. 2010;8:68.

67. Van Dijken JP, Weusthuis RA, Pronk JT. Kinetics of growth and sugar consumption in yeasts. Antonie Van Leeuwenhoek. 1993;63:343-52.

68. Gustin MC, Albertyn J, Alexander M, Davenport K. MAP kinase pathways in the yeast Saccharomyces cerevisiae. Microbiol Mol Biol Rev. 1998;62(4):1264-300. Review. PubMed PMID: 9841672: PubMed Central PMCID: PMC98946.

69. Brewster JL, de Valoir T, Dwyer ND, Winter E, Gustin MC. An osmosensing signal transduction pathway in yeast. Science. 1993;259:1760-3.

70. Kaeberlein M, Andalis AA, Fink GR, Guarente L. High osmolarity extends life span in Saccharomyces cerevisiae by a mechanism related to calorie restriction. Mol Cell Biol. 2002;22(22):8056-66. PubMed PMID: 12391171; PubMed Central PMCID: PMC134739. 